\title{
How to share the cost of cooperating queues in a tandem network?
}

\author{
Judith Timmer and Werner Scheinhardt \\ Department of Applied Mathematics, University of Twente \\ P.O. Box 217, 7500 AE Enschede, The Netherlands \\ E-mail: $\{$ j.b.timmer, w.r.w.scheinhardt\}@utwente.nl
}

\begin{abstract}
We consider networks of queues in which the different operators of individual queues may cooperate to reduce the amount of waiting. More specifically, as a first step we focus on Jackson tandem networks in which the total capacity of the servers can be redistributed over all queues in any desired way. If we associate a cost to waiting that is linear in the queue lengths, it is not difficult to see that the long run total cost is minimized when the operators equally share the available service capacity.

The main question we try to answer is whether or not (the operators of) the individual queues will indeed cooperate in this way, and if so, how they will share the cost in the new situation. We find answers to this question for two- and three-node networks. Keywords: game theory, tandem queue, cooperation, capacity allocation, cost allocation.
\end{abstract}

\section{INTRODUCTION}

Consider a queueing network consisting of different queues, and assume that each of these is operated by a different operator. By working together (in some way), the operators can optimize the performance of the network (in some sense), leading to a social optimum with minimum total cost for the operators. On the other hand, individual operators will try to minimize their own cost, and will only cooperate if this is to their own benefit. This explains our idea of analyzing such networks using cooperative game theory.

In particular, we can view the different operators as decision makers (or players) in a so-called cooperative cost game, see e.g. [7]. In such a game, the players make binding agreements (as opposed to noncooperative games) to jointly optimize the total cost they need to pay, and then try to share this cost by finding a fair cost allocation. Typically, a cost allocation is fair if it lowers the cost for each possible coalition (i.e. for any group of players). If this is not the case, then full cooperation is not beneficial, but there may still be partial cooperation between some (but not all) players.

When we try to model a queueing network as described above, there are a number of choices to be made. First of all, ( $i$ ) one can think of a variety of ways in which the operators may work together, including sharing service capacity, sharing buffer capacity, or changing the routing structure; moreover any of these can be done dynamically or statically (i.e. dependent on the current state of the network or not). Furthermore, (ii) different network topologies may be considered. Similarly, (iii) traffic characteristics, i.e. the behaviour of the arrival process(es) and service demands, can be modeled in many ways. Finally, (iv) the performance of the total network and its individual queues, and the associated cost, can be measured in many ways.

In this paper we study an initial model to investigate whether this line of research is useful to pursue. In this model we make the following assumptions: $(i)$ the different servers are able to share their service capacity, and do so in a static way; ( ii, iii) the network is an $n$-node tandem Jackson network, see e.g. [8]: customers arrive according to a Poisson process at queue 1 , then (after service) move to queue 2, etcetera, until they leave the system (after service at queue $n$ ); service times are all exponentially distibuted; (iv) we take the cost at queue $i$ to be proportional to the long run expected queue length, or equivalently (by Little's law), proportional to the expected sojourn time of customers in queue $i$; furthermore, the total cost of a group of queues is just the sum of the costs of the individual queues (thus, there is no cost associated to the cooperation itself). Even though this model is admittedly very elementary, we find it exhibits some interesting and non-trivial behavior.

In the queueing literature there are many references in which the queues in a network cooperate (sharing capacity, pooling resources, etc.) to reach some form of optimality, e.g. [2], [6]. However, in most cases the whole network is (implicitly) supposed to be run by a single operator. The combination of the queueing model with game theory, in which different operators are only willing to cooperate if a good cost allocation can be 
found, is to the best of our knowledge hardly studied so far. Some related references are the following. González and Herrero [5] study several medical departments that may share an operating theatre. The cost of each department is linear in the capacity needed to satisfy a maximum on the expected waiting time of its patients. It is shown that cooperation reduces the total cost, and that a cost allocation can be determined based on the Shapley value. In [4], García-Sanz et al. extend this model and study cooperation among Markovian queues that share a common server with preemptive priority discipline. The authors show that a cost allocation proportional to the arrival rates is fair. More recently, Anily and Haviv [3] study cooperation in service capacity management. A number of servers pool their capacities and customers. The cost of cooperation is the mean number of customers in the pooled system. It is shown that fair cost allocations always exist. In particular, servers with large capacities may receive payment for cooperation. In these three papers, the servers that cooperate are independent servers. One way in which our paper contributes is that we consider cooperation in a network of queues, while preserving the autonomy of the individual queues; we do not allow for pooling. Finally, we mention Altman et al. [1] who give an extensive survey on networking games. The models and papers discussed in this reference mostly deal with noncooperative game theory, the only exception being a short section focused on bargaining games, which strengthens our belief that the problem formulation and approach in the current paper have not been studied before.

We end this section with a short overview of the remainder of the paper: in Section II we introduce the model in more detail and derive the optimal capacity allocation. Also we recall the basics of cooperative game theory here. Then, in Section III we derive the results on two- and three-node networks, and give some partial results for larger tandem networks. We conclude in Section IV, also sketching some main lines for future research.

\section{MODEL AND PRELIMINARIES}

In this section we first introduce our model in detail, and derive the optimal capacity allocation. Then we recall the basics of cooperative game theory, focusing on the solution concept of the core, which is the set of all cost allocations that are acceptable to all possible coalitions of queues. The last subsection shows how our tandem model fits into the framework of cooperative game theory.

\section{A. Model}

We consider an $n$-node tandem Jackson network, denoting the set of all queues by $N=$ $\{1,2, \ldots, n\}$. Customers arrive at queue 1 according to a Poisson process with rate $\lambda$. The (exponential) service capacity at queue $i$ is given by $\mu_{i}$, and we assume $\mu_{i}>\lambda$. The cost incurred at queue $i$ is represented by the long run expected queue length $\lambda /\left(\mu_{i}-\lambda\right)$. Furthermore, the total cost of any subset $S \subset N$ of queues is the sum of the costs of the individual queues in $S$.

Importantly, we assume that the queues in any subset $S$ may cooperate to improve their performance and save on costs. Cooperation here means that the queues in $S$ may redistribute their service capacities among each other. Denoting the service capacity of queue $i$ after redistribution by $m_{i}$, this leads to the following optimization problem for the set $S$ :

$$
\begin{aligned}
\min _{m_{i}, i \in S} & \sum_{i \in S} \frac{\lambda}{m_{i}-\lambda} \\
\text { s.t. } & \sum_{i \in S} m_{i}=\sum_{i \in S} \mu_{i}, \\
& m_{i}>\lambda, \quad i \in S .
\end{aligned}
$$

To solve this problem, we first consider it without the second constraint, and rewrite it as

$$
\min _{\alpha, m_{i}, i \in S} \sum_{i \in S} \frac{\lambda}{m_{i}-\lambda}-\alpha\left(\sum_{i \in S} m_{i}-\sum_{i \in S} \mu_{i}\right),
$$

where $\alpha$ is the Lagrange multiplier. Taking derivatives leads to the solution

$$
m_{i}=\frac{\sum_{i \in S} \mu_{i}}{|S|} \quad \text { for all } i \in S,
$$

where $|S|$ is the number of queues in $S$. This solution automatically satisfies the second constraint $m_{i}>\lambda$ since $\mu_{i}>\lambda$. Also, since the function $\lambda /(\mu-\lambda)$ is convex in $\mu$, our solution is the optimal capacity allocation. If we denote the average capacity of the queues in subset $S$ by $\bar{\mu}_{S}=$ $\sum_{i \in S} \mu_{i} /|S|$, we can express the corresponding minimal cost for the set $S$ of (cooperating) queues as

$$
c(S)=|S| \frac{\lambda}{\bar{\mu}_{S}-\lambda} .
$$

Notice that in the optimal capacity allocation, capacities are redistributed such that all queues have the same capacity $\bar{\mu}_{S}$ and the same expected number of customers. Consequently, all queues contribute the same amount to the cost for $S$, namely $\lambda /\left(\bar{\mu}_{S}-\lambda\right)$. 


\section{B. Preliminaries on cooperative cost games}

A cooperative cost game is represented by a pair $(N, c)$. The set $N=\{1, \ldots, n\}$ is the set of players. A coalition $S$ is a (nonempty) group of players, that is, a nonempty subset of $N$. The cost function $c$ assigns to each coalition $S$ a certain cost $c(S)$.

In our analysis we will need the concept of marginal vectors, and monotonicity. Let $\sigma$ be a permutation of the player set. Then $\sigma(k)$ is the player in position $k$. Denote by $P_{\sigma}(i)=\{j \in$ $\left.N \mid \sigma^{-1}(j)<\sigma^{-1}(i)\right\}$ the set of players in positions before player $i$. Now imagine that the players enter a room one by one in the ordering indicated by $\sigma$, and that each player has to pay the marginal contribution to the total cost when he enters the room. Then player $i$ pays

$$
m_{i}^{\sigma}=c\left(P_{\sigma}(i) \cup\{i\}\right)-c\left(P_{\sigma}(i)\right) .
$$

The vector $m^{\sigma}=\left(m_{1}^{\sigma}, \ldots, m_{n}^{\sigma}\right)$ is called the marginal vector corresponding to the permutation $\sigma$. Further, a cost game is called monotone increasing (respectively decreasing) if $S \subseteq T$ implies $c(S) \leq c(T)$ (respectively $c(S) \geq c(T)$ ).

If for any two disjoint coalitions $S$ and $T$ of players it is beneficial to cooperate, we say the game is subadditive. In this case, cooperation never leads to higher cost when compared to working separately:

$$
c(S \cup T) \leq c(S)+c(T) .
$$

Remark 1. Notice that in a subadditive game the choice $T=N \backslash S$ in (2) implies $c(N) \leq c(S)+$ $c(N \backslash S)$. Thus, if we split the coalition $N$ of all players in two parts, namely the coalitions $S$ and $N \backslash S$, then the total cost does not decrease. This is an incentive for all the players in coalition $N$ to cooperate.

The main question that remains is how the total joint $\operatorname{cost} c(N)$ should be allocated among the players. A first step towards selecting a good and fair cost allocation is to consider allocations in the core $C(N, c)$ of the game $(N, c)$, which is defined as

$$
\begin{aligned}
C(N, c)= & \left\{y \in \mathbb{R}^{N} \mid \sum_{i \in N} y_{i}=c(N) ;\right. \\
& \left.\sum_{i \in S} y_{i} \leq c(S) \text { for all } S \subset N\right\} .
\end{aligned}
$$

If the cost is allocated among the players according to an allocation in the core, then any coalition $S$ pays at most its own costs $c(S)$. Hence, no coalition has an incentive to break up the cooperation with coalition $N$.

We are now ready to view the tandem queue problem as a cooperative cost game.

\section{Tandem games}

Based on the optimal capacity allocation of a group of queues in our $n$-node tandem Jackson network, see Subsection II-A, we can now define a corresponding cooperative cost game. From now on we refer to this game as a tandem game.

Definition. A tandem game is a cost game $(N, c)$ with the set of queues $N=\{1, \ldots, n\}$ as player set. The cost $c(S)$ of coalition $S \subseteq N$ is given by (1).

\section{Proposition 1. The following properties hold.}

(i) Tandem games are not monotone decreasing, unless all capacities $\mu_{i}$ are equal.

(ii) For all $n \geq 2$, $n$-node tandem games may or may not be monotone increasing.

(iii) Tandem games are subadditive.

Proof. For (i), assume without loss of generality that $\mu_{1}<\mu_{2}$, and let $T=\{1,2\}$ and $S=\{2\}$. Then $\bar{\mu}_{T}<\mu_{2}$ immediately implies $c(S)=$ $\lambda /\left(\mu_{2}-\lambda\right)<2 \lambda /\left(\bar{\mu}_{T}-\lambda\right)=c(T)$.

To prove (ii), we construct two concrete examples. A (non-trivial) example of an $n$-node monotone increasing tandem game can be found by choosing the capacities sufficiently close together, e.g. take $\lambda=1$ and all $\mu_{i}$ inside the interval $[2-\varepsilon, 2+\varepsilon]$ for some $\varepsilon$. Then the cost of any $k$-node coalition lies inside $[k /(1+\varepsilon), k /(1-\varepsilon)]$. By taking $\varepsilon<1 /(2 k+1)$ we can ensure that these intervals do not overlap for different $k \leq n$. On the other hand, any $n$-node tandem game with $\mu_{2}>3 \mu_{1}-2 \lambda$ is not monotone increasing since for $T=\{1,2\}$ we have $c(T)=2 \lambda /\left(\bar{\mu}_{T}-\lambda\right)<\lambda /\left(\mu_{1}-\lambda\right)=c(\{1\})$.

To prove (iii) we substitute (1) in (2), which leads to

$$
\frac{\lambda}{\bar{\mu}_{S \cup T}-\lambda} \leq \frac{|S|}{|S|+|T|} \frac{\lambda}{\bar{\mu}_{S}-\lambda}+\frac{|T|}{|S|+|T|} \frac{\lambda}{\bar{\mu}_{T}-\lambda} .
$$

Since

$$
\bar{\mu}_{S \cup T}=\frac{|S|}{|S|+|T|} \bar{\mu}_{S}+\frac{|T|}{|S|+|T|} \bar{\mu}_{T},
$$

the result follows by the convexity of $\lambda /(\mu-\lambda)$ as a function of $\mu$.

The fact that tandem queues are usually not monotone is not helpful for the analysis of the core. Before we move on to this in Section III, we 
present some weaker monotonicity results. In the following proposition we show that the total cost of any coalition may increase or decrease by adding queues with sufficiently low or high capacity to the coalition.

Proposition 2. Consider two coalitions $S$ and $T$ satisfying $S \subseteq T \subseteq N$. Then $c(S) \leq c(T)$ is equivalent with

$$
\bar{\mu}_{T \backslash S} \leq \bar{\mu}_{S}+\frac{|T|}{|S|}\left(\bar{\mu}_{S}-\lambda\right) .
$$

In the particular case where $T \backslash S$ only contains a single node, say $T=S \cup\{i\}$ with $i \notin S$, we have the following.

(i) A simple sufficient condition for $c(S) \leq c(T)$ (increasing cost when adding queue $i$ to $S$ ) is given by $\mu_{i} \leq \bar{\mu}_{S}$.

(ii) When $|S|>2$ a simple sufficient condition for $c(S)>c(T)$ (decreasing cost when adding queue $i$ to $S)$ is given by $\mu_{i}>$ $\sum_{j \in S} \mu_{j}$.

Proof. Using (1) we have that $c(S) \leq c(T)$ is equivalent to

$$
\frac{|S|}{\bar{\mu}_{S}-\lambda} \leq \frac{|T|}{\bar{\mu}_{T}-\lambda} .
$$

Since we also know that

$$
\begin{aligned}
\bar{\mu}_{T} & =\frac{|S| \bar{\mu}_{S}+(|T|-|S|) \bar{\mu}_{T \backslash S}}{|T|} \\
& =\bar{\mu}_{S}+\frac{|T|-|S|}{|T|}\left(\bar{\mu}_{T \backslash S}-\bar{\mu}_{S}\right),
\end{aligned}
$$

this can be rewritten as

$|S|\left(\bar{\mu}_{S}+\frac{|T|-|S|}{|T|}\left(\bar{\mu}_{T \backslash S}-\bar{\mu}_{S}\right)-\lambda\right) \leq|T|\left(\bar{\mu}_{S}-\lambda\right)$.

Solving for $\bar{\mu}_{T \backslash S}$ leads to (3).

When $T=S \cup\{i\}$ with $i \notin S$, we have $\bar{\mu}_{T \backslash S}=$ $\mu_{i}$. Statement $(i)$ is then immediate. For statement (ii) we need to show that

$$
\sum_{j \in S} \mu_{j}>\bar{\mu}_{S}+\frac{|S|+1}{|S|}\left(\bar{\mu}_{S}-\lambda\right)
$$

holds when $|S|>2$. Dividing by $\bar{\mu}_{S}$ yields

$$
|S|>1+\frac{|S|+1}{|S|}\left(1-\frac{\lambda}{\bar{\mu}_{S}}\right),
$$

or

$$
1-\frac{\lambda}{\bar{\mu}_{S}}<\frac{|S|(|S|-1)}{|S|+1},
$$

which is indeed true when $|S|>2$ since the righthand side is then larger than 1 while the left-hand side is less than 1 .
Remark 2. For a two-node tandem queue with $\mu_{1}<\mu_{2}$, the first part of this proposition enables us to deduce that the game is monotone increasing if and only if $\mu_{1} \geq \frac{1}{3} \mu_{2}+\frac{2}{3} \lambda$. See also the proof of Proposition 1, part (ii).

Remark 3. Notice that in all of the above we consider whether the total cost of a coalition increases or decreases by adding other queues. If we want to compare the cost per queue, the situation is less complicated: the average cost per queue will simply increase (respectively decrease) if we add queues to $S$ with average capacity that is smaller (respectively higher) than $\bar{\mu}_{S}$.

As mentioned before, in the next section we study the core of tandem games. In particular we want to know if fair cost allocations always exist. That is, if the core is always a nonempty set.

\section{MAIN RESULTS}

In this section we first derive results on the core of two- and three-node networks. Then we point out how larger networks differ from three-node networks.

\section{A. Two-node tandem games}

For two-node tandem games, we have the following result.

Theorem 3. Tandem games corresponding to twonode networks have a nonempty core.

Proof. This follows immediately from the subadditivity property (2) of tandem games, see Remark 1.

We give an example to illustrate that the core can easily be found explicitly in this case.

Example 1. Consider a two-node network with arrival rate $\lambda=1 / 4$ and service rates $\left(\mu_{1}, \mu_{2}\right)=$ $(1 / 3,2 / 3)$. The costs of the coalitions are $c(\{1\})=$ $3, c(\{2\})=3 / 5$, and $c(N)=2$. By Theorem 3 cooperation is worthwhile since cost savings are achieved: $c(N)<c(\{1\})+c(\{2\})$. The core of the game is nonempty and equals

$$
C(N, c)=\{(x, 2-x) \mid 7 / 5 \leq x \leq 3\} .
$$

Notice that in this example there exist core allocations such that the cost allocated to the second queue is negative, for example $(3,-1)$. In such allocations the second queue gets paid for its cooperation. Note that the second queue has the largest capacity; its contribution is so valuable that this queue may receive payment to cooperate. 
This phenomenon is not always present, e.g. when $\left(\mu_{1}, \mu_{2}\right)=(2 / 5,3 / 5)$, we have $c(\{1\})=5 / 3$, $c(\{2\})=5 / 7, c(N)=2$ and $C(N, c)=$ $\{(x, 2-x) \mid 9 / 7 \leq x \leq 5 / 3\}$.

\section{B. Three-node tandem games}

A similar result holds for three-node networks. For these networks we identify two cost allocations that belong to the core.

Theorem 4. Tandem games corresponding to three-node networks have a nonempty core. In particular, if $\sigma$ is a permutation such that the queue with middle service capacity ${ }^{1}$ is in first position, then the marginal vector $m^{\sigma}(c)$ is a cost allocation that belongs to the core of the game.

Proof. Without loss of generality assume that $\mu_{1} \geq$ $\mu_{2} \geq \mu_{3}$. Then queue 2 is the queue with middle service capacity. There are two marginal vectors in which node 2 is in first position, $\sigma(1)=2$, namely $m^{213}(c)$ and $m^{231}(c)$. Here we denote $\sigma$ by $\sigma(1) \sigma(2) \sigma(3)$. Consider the first marginal vector. The marginal contributions of the nodes are

$$
\begin{aligned}
& m_{1}^{213}(c)=c(\{1,2\})-c(\{2\}) \\
& m_{2}^{213}(c)=c(\{2\}) \\
& m_{3}^{213}(c)=c(N)-c(\{1,2\}) .
\end{aligned}
$$

We proceed by checking the core-conditions:

- $m_{1}^{213}(c) \leq c(\{1\})$ : true by subadditivity;

- $m_{2}^{213}(c) \leq c(\{2\})$ : true (with equality);

- $m_{3}^{213}(c) \leq c(\{3\})$ : true by subadditivity;

- $m_{1}^{213}(c)+m_{2}^{213}(c) \leq c(\{1,2\})$ : true (with equality);

- $m_{1}^{213}(c)+m_{3}^{213}(c) \leq c(\{1,3\})$ : true by subadditivity;

- $m_{2}^{213}(c)+m_{3}^{213}(c) \leq c(\{2,3\})$ : see below;

- $m_{1}^{213}(c)+m_{2}^{213}(c)+m_{3}^{213}(c)=c(N)$ : true.

The condition related to coalition $\{2,3\}$ is

$$
c(\{2\})+c(N)-c(\{1,2\}) \leq c(\{2,3\}) .
$$

This can be shown by straightforward calculations:

$$
\begin{aligned}
c(N)+ & c(\{2\}) \\
\leq & \frac{\lambda}{\bar{\mu}_{\{1,2\}}-\lambda}+\frac{\lambda}{\bar{\mu}_{\{2,3\}}-\lambda}+\frac{\lambda}{\bar{\mu}_{\{1,3\}}-\lambda} \\
& +\frac{\lambda}{\mu_{2}-\lambda}
\end{aligned}
$$

\footnotetext{
${ }^{1}$ If two or three queues have equal capacity, either of these
} can be chosen as 'the' queue with middle service capacity.

$$
\begin{aligned}
\leq & \frac{\lambda}{\bar{\mu}_{(\{1,2\}}-\lambda}+\frac{\lambda}{\bar{\mu}_{\{2,3\}}-\lambda}+\frac{\lambda}{\bar{\mu}_{\{1,2\}}-\lambda} \\
& +\frac{\lambda}{\bar{\mu}_{\{2,3\}}-\lambda} \\
= & \frac{2 \lambda}{\bar{\mu}_{\{1,2\}}-\lambda}+\frac{2 \lambda}{\bar{\mu}_{\{2,3\}}-\lambda} \\
= & c(\{1,2\})+c(\{2,3\}) .
\end{aligned}
$$

The second inequality follows from the convexity of $\lambda /(\mu-\lambda)$ in $\mu$.

For the second marginal vector $m^{231}(c)$, the marginal contributions of the nodes are

$$
\begin{aligned}
& m_{1}^{213}(c)=c(N)-c(\{2,3\}) \\
& m_{2}^{213}(c)=c(\{2\}), \\
& m_{3}^{213}(c)=c(\{2,3\})-c(\{2\}) .
\end{aligned}
$$

As above, the core-conditions can be checked to hold, either with equality, or by subadditivity. The only exception is the condition related to coalition $\{1,2\}: c(N)-c(\{2,3\})+c(\{2\}) \leq c(\{1,2\})$, which is equivalent to (4) and therefore also holds. This proves the result.

The theorem above shows that also three-node tandem games have a nonempty core. Notice that any convex combination of the two abovementioned marginal vectors also belongs to the core because this is a convex set.

Cost allocations in the core of three-node tandem games may have negative elements - the corresponding queue receives payment to cooperate - as was the case for two-node networks. For example, consider the situation in Example 1, and add a third queue with capacity $\mu_{3}=1 / 3$. According to Theorem 4 , the cost allocation $m^{123}(c)=$ $(3,-1,13 / 7)$ belongs to the core of the game. Node 2 has the largest service capacity, and may be paid for cooperation.

The two particular cost allocations identified in Theorem 4 are such that the queue with middle service capacity does not gain from cooperation. That is, the cost that is allocated to this queue is the same as its stand-alone cost $c(\{i\})$. Below we characterize the conditions under which all cost allocations in the core have this property.

Proposition 5. Consider a three-node network. Let queиe $i$ be the queue with middle service capacity. If this capacity is equal to the average capacity of the other queues, $\mu_{i}=\sum_{j \neq i} \mu_{j} / 2$, then queue $i$ does not gain from cooperation: $x_{i}=c(\{i\})$ for all cost allocations $x$ in the core $C(N, c)$ of the tandem game. 
Proof. Without loss of generality let $i=2$ be the queue with middle service capacity. Let $x$ be a cost allocation in the core $C(N, c)$. By definition of the core, this allocation satisfies the restrictions $x_{2} \leq c(\{2\}), x_{1}+x_{3} \leq c(\{1,3\})$, and $x_{1}+x_{2}+$ $x_{3}=c(N)=c(\{2\})+c(\{1,3\})$ (where the latter equality follows from $\left.\mu_{2}=\left(\mu_{1}+\mu_{3}\right) / 2\right)$. Combining these restrictions results in $x_{2}=c(\{2\})$.

Remark 4. One may be inclined to argue that when a player does not gain from cooperation, as in the context of Proposition 5, he may refrain from cooperation to prevent extra benefit for the other players. However, it is easy to see that his decision to cooperate or not does not affect the cost paid (or amount gained) by the other players. The reason is that his service capacity equals the average capacity of all (other) players, and that the cost paid by the other (cooperating) players is based on this same average capacity.

\section{Tandem games with more than three nodes}

It is not easy to obtain results for $n$-node tandem Jackson networks with $n>3$. If all queues have equal service capacities, then we obtain the following obvious result.

Proposition 6. Consider an n-node tandem Jackson network in which all queues have equal capacity, $\mu_{i}=\mu$ for all $i$. Then the core of the corresponding tandem game consists of a single allocation $x$ with $x_{i}=\lambda /(\mu-\lambda)$ for all $i$.

Proof. Let $S$ be a coalition of queues. The cost of this coalition is $c(S)=|S| \lambda /(\mu-\lambda)$. The corresponding tandem game has the property $c(S)=$ $\sum_{i \in S} c(\{i\})$. Combining this property with the definition of the core proves the proposition.

For three-node tandem networks we showed that the core is nonempty by identifying two marginal vectors that belong to the core, see Theorem 4. This approach cannot be extended to four-node networks, as the following example illustrates.

Example 2. Consider the four-node tandem network with arrival rate $\lambda=1$, and service rates $\mu_{1}=8, \mu_{2}=6, \mu_{3}=4$ and $\mu_{4}=2$. The costs of the various coalitions in the corresponding tandem game are given in Table I. The 24 marginal vectors are listed in Table II.

One can verify that none of these marginal vectors belongs to the core of the game. However, the core of the game is nonempty since, for example, the allocation $x=(15,180,315,480) / 990$ belongs to the core.

We need a different approach to study the core of the four-node tandem game. Further research is needed to determine if cooperation is also beneficial for tandem networks with at least four nodes.

\section{CONCLUSIONS AND FUTURE WORK}

\section{A. Conclusions}

For the particular model we analyzed, so far we found the following partial answers to the question if and how cooperation will take place:

- In two-node networks, cooperation will always take place, and there are many ways to share the cost such that the new situation is preferred by both operators. Interestingly, it may happen (depending on parameters) that the queue with largest capacity will have negative cost, in other words it may be paid by the other queue for the cooperation.

- In three-node networks, cooperation will always take place, since cost allocations exist such that any combination (or coalition) of queues has lower (or equal) total cost than in the case without cooperation. One class of cost allocations is such that the queue with 'middle' capacity will pay the same cost as without cooperation.

- In networks with more than three nodes, it is not clear whether a cost allocation (always) exists that satisfies all possible coalitions. Case studies suggest that cooperation is still beneficial for the four-queue case.

\section{B. Future work}

As mentioned in the introduction, many assumptions can be done within our framework of 'queueing network games'. In particular we intend to further develop the current model, leading to a complete characterization of the cases that may occur for the general $n$-node tandem model. If this is feasible, some of the results can probably be generalized to a more general (Jackson) network topology.

Another way of "sharing capacity" would be to change the routing pattern such that 'underloaded' queues can provide service to jobs that otherwise would have been routed to other (highly loaded) queues.

Finally, it would also be interesting to consider dynamic ways of cooperation, and/or stochastic cost structures. 


\begin{tabular}{|r|cccc|cccccc|}
\hline $\mathrm{S}$ & $\{1\}$ & $\{2\}$ & $\{3\}$ & $\{4\}$ & $\{1,2\}$ & $\{1,3\}$ & $\{1,4\}$ & $\{2,3\}$ & $\{2,4\}$ & $\{3,4\}$ \\
$\mathrm{c}(\mathrm{S})$ & $1 / 7$ & $1 / 5$ & $1 / 3$ & 1 & $1 / 3$ & $2 / 5$ & $1 / 2$ & $1 / 2$ & $2 / 3$ & 1 \\
\hline $\mathrm{S}$ & $\{1,2,3\}$ & $\{1,2,4\}$ & $\{1,3,4\}$ & $\{2,3,4\}$ & $\{1,2,3,4\}$ & & & & & \\
$\mathrm{c}(\mathrm{S})$ & $3 / 5$ & $9 / 13$ & $9 / 11$ & 1 & 1 & & & & & \\
\hline
\end{tabular}

TABLE I

COSTS OF ALL COALITIONS FOR THE GAME IN EXAMPLE 2.

\begin{tabular}{|llllll|llllll|}
\hline$m^{1234}$ & $m^{1243}$ & $m^{1324}$ & $m^{1342}$ & $m^{1423}$ & $m^{1432}$ & $m^{2134}$ & $m^{2143}$ & $m^{2314}$ & $m^{2341}$ & $m^{2413}$ & $m^{2431}$ \\
\hline 0.143 & 0.143 & 0.143 & 0.143 & 0.143 & 0.143 & 0.133 & 0.133 & 0.100 & 0 & 0.026 & 0 \\
0.190 & 0.190 & 0.400 & 0.182 & 0.192 & 0.182 & 0.200 & 0.200 & 0.200 & 0.200 & 0.200 & 0.200 \\
0.267 & 0.308 & 0.257 & 0.257 & 0.308 & 0.318 & 0.267 & 0.308 & 0.300 & 0.300 & 0.308 & 0.333 \\
0.400 & 0.359 & 0.200 & 0.418 & 0.357 & 0.357 & 0.400 & 0.359 & 0.400 & 0.500 & 0.467 & 0.467 \\
\hline
\end{tabular}

\begin{tabular}{|llllll|llllll|}
\hline$m^{3124}$ & $m^{3142}$ & $m^{3214}$ & $m^{3241}$ & $m^{3412}$ & $m^{3421}$ & $m^{4123}$ & $m^{4132}$ & $m^{4213}$ & $m^{4231}$ & $m^{4312}$ & $m^{4321}$ \\
\hline 0.067 & 0.067 & 0.100 & 0 & -0.182 & 0 & -0.500 & -0.500 & 0.026 & 0 & -0.182 & 0 \\
0.200 & 0.182 & 0.167 & 0.167 & 0.182 & 0 & 0.192 & 0.182 & -0.333 & -0.333 & 0.182 & 0 \\
0.333 & 0.333 & 0.333 & 0.333 & 0.333 & 0.333 & 0.308 & 0.318 & 0.308 & 0.333 & 0 & 0 \\
0.400 & 0.418 & 0.400 & 0.500 & 0.667 & 0.667 & 1 & 1 & 1 & 1 & 1 & 1 \\
\hline
\end{tabular}

TABLE II

THE 24 MARGINAL VECTORS OF THE GAME IN EXAMPLE 2. TOP TO BOTTOM ENTRIES CORRESPOND TO PLAYERS 1 TILL 4.

\section{REFERENCES}

[1] E. Altman, T. Boulogne, R. El-Azouzi, T. Jiménez, and L. Wynter. A survey on networking games in telecommunications. Computers \& Operations Research, 33(2):286 311, 2006.

[2] S. Andradóttir and H. Ayhan. Throughput maximization for tandem lines with two stations and flexible servers. Operations Research, 53:516-531, 2005.

[3] S. Anily and M. Haviv. Cooperation in service systems. Operations Research. (To appear.) Online available at http://pluto.huji.ac.il/ haviv/quegam44N.pdf.
[4] M.D. García-Sanz, F.R. Fernández, M.G. Fiestras-Janeiro, I. García-Jurado, and J. Puerto. Cooperation in Markovian queueing models. European J. Oper. Res., 188(2):485-495, 2008.

[5] P. González and C. Herrero. Optimal sharing of surgical costs in the presence of queues. Math. Methods Oper. Res., 59(3):435-446, 2004.

[6] A. Mandelbaum and M.J. Reiman. On pooling in queueing networks. Management Science, 44:971-981, 1998.

[7] H. Peters. Game theory. Springer-Verlag, Berlin, 2008.

[8] J. Walrand. An introduction to queueing networks. PrenticeHall, New Jersey, 1988. 